\title{
ANZTLA 2004 Statistics
}

\section{compiled by Kerrie Stevens}

\author{
Notes on ANZTLA 2004 Statistics
}

\section{Reporting Categories:}

A Provides clergy training programmes, (i.e. ANZATS member schools) and/or schools and training institutions which offer broad theological training (i.e. Bible Colleges, Missionary Training Institutions) possibly leading to degree or post-graduate qualifications

B Do not have students (i.e. church administrative libraries, resource centres, para-church organizations, etc.)

C Institutions offering non-theological courses in addition (i.e. teacher training)

\section{Statistics Response:}

Of the 108 questionnaires sent out, 49 , or $52.9 \%$ were returned. This is down from last year $(55.67 \%)$

\section{Future Statistics Questionnaires:}

The statistics questionnaire will be reviewed in the second half of 2005, and distributed in early 2006 for 2005 data.

Thank you to all the Libraries who responded with their statistics!

Kerrie Stevens

Statistician 
TABLE 1A 2004 LIBRARY STAFF

\begin{tabular}{|c|c|c|c|c|c|}
\hline TINSTIIUTION & STATEINZ & $\begin{array}{l}\text { PROFESIONAL } \\
\text { [1] }\end{array}$ & $\begin{array}{l}\text { PARA-PROFESSIONAL } \\
{[2]}\end{array}$ & $\begin{array}{l}\text { LIBRARY } \\
\text { SUPPORT } \\
{[3]}\end{array}$ & $\begin{array}{l}\text { TOTAL } \\
\text { STAFF } \\
\text { [4] }\end{array}$ \\
\hline Adelaide College of Divinity & SA & 1.6 & 0 & 0.58 & 2.18 \\
\hline Alliance College of Australia & ACT & 1.00 & 0.00 & 0.00 & 1.00 \\
\hline Australian Lutheran College & SA & 2.60 & 0.00 & 1.20 & 3.80 \\
\hline Bible College of New Zealand & $\mathrm{NZ}$ & 2.00 & 1.00 & 0.50 & 3.50 \\
\hline Bible College of Victoria & VIC & 1.00 & 0.00 & 0.30 & 1.30 \\
\hline Booth College of Mission & $\mathrm{NZ}$ & 1.00 & 0.00 & 0.00 & 1.00 \\
\hline Bible College of South Australia & SA & 1.00 & 0.00 & 0.00 & 1.00 \\
\hline Burleigh College & SA & 0.40 & 0.00 & 0.20 & 0.60 \\
\hline Camden Theological Library & NSW & 2.00 & 0.80 & 0.00 & 2.80 \\
\hline Carey Baptist College & NZ & 2.00 & 1.00 & 0.00 & 3.00 \\
\hline Catholic Theological College & VIC & 1.80 & 1.00 & 0.00 & 2.80 \\
\hline Churches of Christ Theological College & VIC & 0.20 & 0.00 & 0.00 & 0.20 \\
\hline Garden City College of Ministries & QLD & 0.40 & 0.00 & 0.00 & 0.40 \\
\hline Harvest Bible College & $\mathrm{VIC}$ & 1.00 & 0.00 & 0.00 & 1.00 \\
\hline Joint Theological Library & VIC & 2.50 & 1.60 & 0.00 & 4.10 \\
\hline Kingsley College & $\mathrm{VIC}$ & 1.00 & 0.00 & 0.00 & 1.00 \\
\hline Moore Theological College & NSW & 3.00 & 3.00 & 1.00 & 7.00 \\
\hline Nazarene Theological College & QLD & 0.67 & 0.22 & 0.16 & 1.05 \\
\hline New Creation Library & SA & 0.00 & 0.00 & 3.00 & 3.00 \\
\hline Phlair International College & $\mathrm{NZ}$ & 1.00 & 0.00 & 2.00 & 3.00 \\
\hline Presbyterian Theological Centre & NSW & 0.80 & 0.00 & 0.00 & 0.80 \\
\hline Presbyterian Theological College & VIC & 0.50 & 0.00 & 0.00 & 0.50 \\
\hline Queensland Baptist College of Ministries & QLD & 0.60 & 0.00 & 0.00 & 0.60 \\
\hline Ridley College & VIC & 1.31 & 0.00 & 0.35 & 1.66 \\
\hline St. Andrew's Greek Orthodox Theological Coll. & NSW & 1.00 & 0.00 & 0.30 & 1.30 \\
\hline St. Charles Seminary & WA & 0.60 & 0.00 & 0.00 & 0.60 \\
\hline St. Francis' Theological College & QLD & 1.00 & 0.00 & 0.33 & 1.33 \\
\hline St. John's College Ministry Centre & NSW & 0.00 & 0.60 & 0.00 & 0.60 \\
\hline St. Paschal Library & VIC & 1.80 & 0.00 & 1.00 & 2.80 \\
\hline Sydney Missionary \& Bible College & NSW & 1.40 & 0.20 & 0.00 & 1.60 \\
\hline Tabor College - Victoria & $\mathrm{VIC}$ & 1.00 & 0.00 & 1.30 & 2.30 \\
\hline Tahlee Bible College & NSW & 0.40 & 0.00 & 0.50 & 0.90 \\
\hline Talua Ministry Training Centre & VAN & 1.00 & 0.00 & 1.00 & 2.00 \\
\hline Trinity Theological College & WA & 0.80 & 0.20 & 0.20 & 1.20 \\
\hline Vianney College & NSW & 0.03 & 0.00 & 0.00 & 0.03 \\
\hline Wesley Institute & NSW & 1.00 & 0.60 & 0.40 & 2.00 \\
\hline Worldview Centre for Intercultural Studies & TAS & 0.20 & 0.60 & 0.30 & 1.10 \\
\hline TOTAL & & 39.61 & 10.82 & 14.62 & 65.05 \\
\hline AVERAGE & & 1.07 & 0.29 & 0.40 & 1.76 \\
\hline MEDIAN & & 1.00 & 0.00 & 0.16 & 1.30 \\
\hline
\end{tabular}

VAN $=$ Vanuatu 
TABLE 1B 2004 LIBRARY STAFF

\begin{tabular}{|c|c|c|c|c|c|}
\hline TINSTITUTION & STATEINZ & $\begin{array}{c}\text { PROFESSIONAL } \\
\text { [1] }\end{array}$ & $\begin{array}{c}\text { PARA- } \\
\text { PROFESSIONAL } \\
\text { [2] }\end{array}$ & $\begin{array}{c}\text { LIBRARY } \\
\text { SUPPORT } \\
\text { [3] }\end{array}$ & $\begin{array}{l}\text { TOTAL } \\
\text { STAFF } \\
\text { [4] }\end{array}$ \\
\hline Benedictine Community of New Norcia & WA & 0.60 & 0.90 & 0.50 & 2.00 \\
\hline Carmelite Friars & VIC & 0.50 & 0.40 & 0.00 & 0.90 \\
\hline Catholic Education Office & SA & 1.00 & 0.00 & 1.00 & 2.00 \\
\hline Catholic Education Office & WA & 2.00 & 2.00 & 2.00 & 6.00 \\
\hline Dominican Fathers & $\mathrm{VIC}$ & 0.80 & 0.20 & 0.00 & 1.00 \\
\hline Evanelical Library & SA & 0.00 & 0.00 & 0.00 & 0.00 \\
\hline St. Benedict's Monastery & NSW & 0.20 & 0.20 & 0.00 & 0.40 \\
\hline TOTAL & & 5.10 & 3.70 & 3.50 & 12.30 \\
\hline AVERAGE & & 0.73 & 0.53 & 0.50 & 1.76 \\
\hline MEDIAN & & 0.60 & 0.20 & 0.00 & 1.00 \\
\hline
\end{tabular}

TABLE 1C 2004 LIBRARY STAFF

\begin{tabular}{|c|c|c|c|c|c|}
\hline TINSTITUTION & STATEINZ & $\begin{array}{c}\text { PROFESSIONAL } \\
\text { [1] }\end{array}$ & $\begin{array}{c}\text { PARA- } \\
\text { PROFESSIONAL } \\
\text { [2] }\end{array}$ & $\begin{array}{c}\text { LIBRARY } \\
\text { SUPPORT } \\
\text { [3] }\end{array}$ & $\begin{array}{c}\text { TOTAL. } \\
\text { STAFF } \\
\text { [4] }\end{array}$ \\
\hline Australian Catholic University - Banyo & QLD & 6.12 & 6.70 & 2.80 & 15.62 \\
\hline Australian Catholic University - Signadou & ACT & 3.00 & 1.60 & 1.50 & 6.10 \\
\hline $\begin{array}{l}\text { Australian Catholic University - St. } \\
\text { Patrick's }\end{array}$ & $\mathrm{VIC}$ & 6.00 & 6.60 & 4.80 & 17.40 \\
\hline MASTERS Institute & $\mathrm{NZ}$ & 0.00 & 1.00 & 0.00 & 1.00 \\
\hline $\begin{array}{l}\text { Trínity College - Leeper \& Mollison } \\
\text { Libraries }\end{array}$ & VIC & 2.2 & 0 & 0.4 & 2.60 \\
\hline University of Notre Dame & WA & 0.5 & 0 & 0 & 0.50 \\
\hline TOTAL & & 17.82 & 15.90 & 9.50 & 43.22 \\
\hline AVERAGE & & 2.97 & 2.65 & 1.58 & 7.20 \\
\hline MEDIAN & & 2.60 & 1.30 & 0.95 & 4.35 \\
\hline
\end{tabular}


TABLE 2A 2004 LIBRARY SERVICES

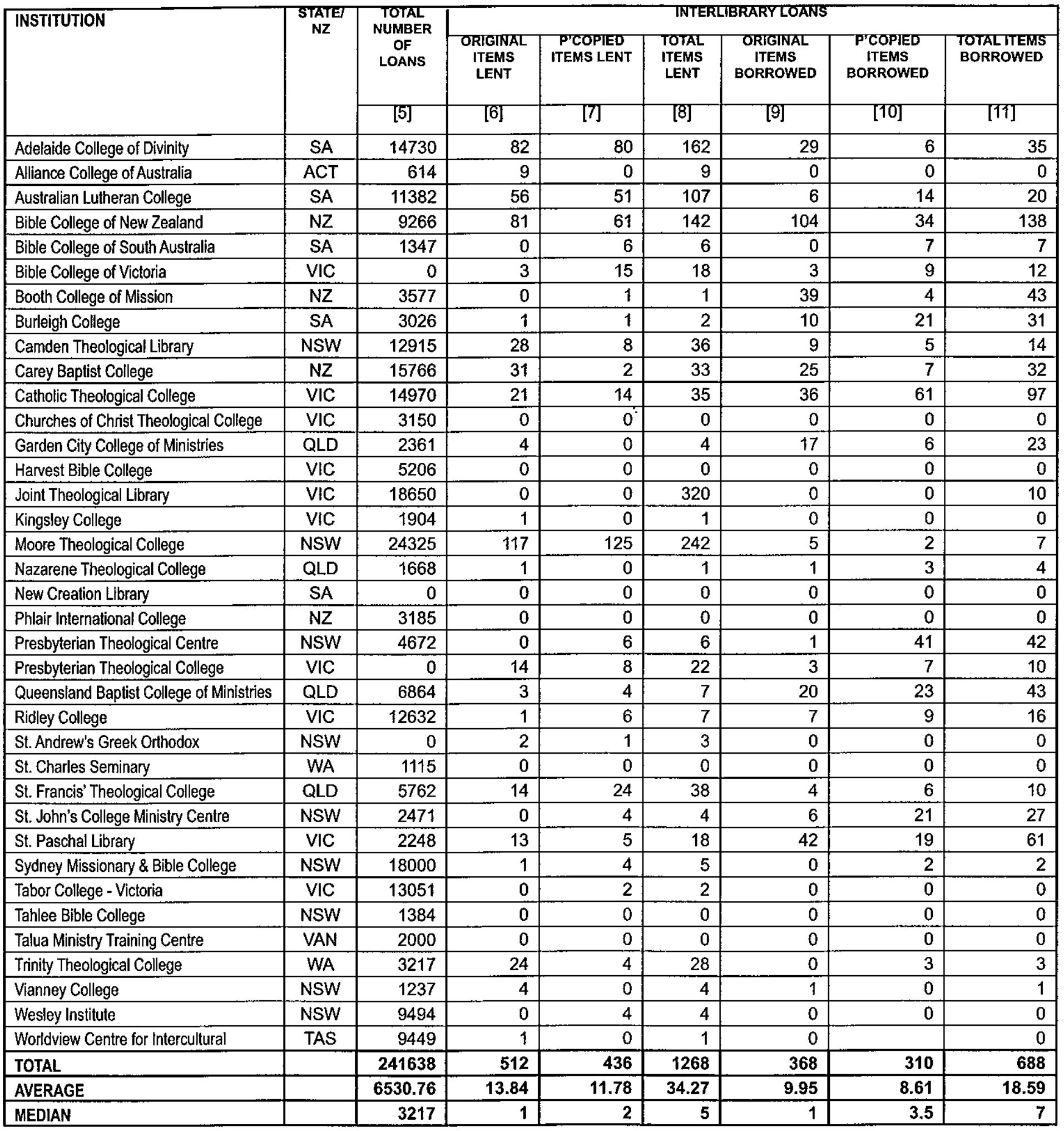


TABLE 2B 2004 LIBRARY SERVICES

\begin{tabular}{|c|c|c|c|c|c|c|c|c|}
\hline \multirow[t]{3}{*}{ TNSTITUTION } & \multirow{3}{*}{$\begin{array}{l}\text { STATE } \\
\text { / NZ }\end{array}$} & \multirow{2}{*}{$\begin{array}{c}\text { TOTAL } \\
\text { NUMBER } \\
\text { OF } \\
\text { LOANS }\end{array}$} & \multicolumn{6}{|c|}{ TNTERLIBRARY LOANS } \\
\hline & & & $\begin{array}{l}\text { ORIGINAL } \\
\text { ITEMS } \\
\text { LENT }\end{array}$ & & $\begin{array}{l}\text { TOTAL } \\
\text { ITEMS } \\
\text { LENT }\end{array}$ & $\begin{array}{c}\text { ORIGINAL } \\
\text { ITEMS } \\
\text { BORROWED }\end{array}$ & $\begin{array}{l}\text { P'COPIED } \\
\text { ITEMS } \\
\text { BORROWED }\end{array}$ & $\begin{array}{l}\text { TOTAL ITEMS } \\
\text { BORROWED }\end{array}$ \\
\hline & & [5] & [6] & [7] & $78]$ & [9] & {$[10]$} & [11] \\
\hline Benedictine Community of New Norcia & WA & 100 & 0 & 10 & 10 & 0 & 0 & 0 \\
\hline Carmelite Friars & VIC & 546 & 6 & 11 & 17 & 0 & 0 & 0 \\
\hline Catholic Education Office & SA & 12676 & 0 & 0 & 0 & 0 & 0 & 0 \\
\hline Catholic Education Office & WA & 26645 & 0 & 0 & 0 & 0 & 0 & 0 \\
\hline Dominican Fathers & VIC & 2000 & 4 & 6 & 10 & 0 & 4 & 4 \\
\hline Evangelica Library & SA & 4186 & 0 & 0 & 0 & 0 & 0 & 0 \\
\hline St. Benedict's Monastery & NSW & 0 & 1 & 3 & 4 & 1 & 0 & 1 \\
\hline TOTAL & & 46153 & 11 & 30 & 41 & 1 & 4 & 5 \\
\hline AVERAGE & & 6593.29 & 1.57 & 4.29 & 5.86 & 0.14 & 0.57 & 0.71 \\
\hline MEDIAN & & 2000.00 & 0.00 & 3.00 & 4.00 & 0.00 & 0.00 & 0.00 \\
\hline
\end{tabular}

TABLE 2C 2004 LIBRARY SERVICES

\begin{tabular}{|c|c|c|c|c|c|c|c|c|}
\hline \multirow[t]{3}{*}{ TINSTITUTION } & \multirow{3}{*}{$\begin{array}{l}\text { STATE } \\
\text { INZ }\end{array}$} & \multirow{2}{*}{$\begin{array}{c}\text { TOTAL } \\
\text { NUMBER } \\
\text { OF } \\
\text { LOANS }\end{array}$} & \multicolumn{6}{|c|}{ TNTERLIBRARYLOANS } \\
\hline & & & $\begin{array}{l}\text { ORIGINAL } \\
\text { ITEMS } \\
\text { LENT }\end{array}$ & & $\begin{array}{l}\text { TOTAL } \\
\text { ITEMS } \\
\text { LENT }\end{array}$ & $\begin{array}{l}\text { ORIGINAL } \\
\text { ITEMS } \\
\text { BORROWED }\end{array}$ & & $\begin{array}{l}\text { TOTAL ITEMS } \\
\text { BORROWED }\end{array}$ \\
\hline & & [5] & [6] & {$[7]$} & [8] & [9] & {$[10]$} & [11] \\
\hline Australian Catholic University - Banyo & QLD & 74155 & 771 & 227 & 998 & 344 & 635 & 979 \\
\hline Australian Catholic University - Signadou & ACT & 30485 & 246 & 56 & 302 & 193 & 102 & 295 \\
\hline MASTERS Institute & NZ & 6283 & 0 & 0 & 0 & 0 & 0 & 0 \\
\hline Trinity College - Leeper \& Mollison Libraries & VIC & 10607 & 0 & 0 & 0 & 2 & 0 & 2 \\
\hline University of Notre Dame* & WA & 0 & 0 & 0 & 0 & 0 & 0 & 0 \\
\hline TOTAL & & 202914 & 1349 & 466 & 1815 & 663 & 1103 & 1766 \\
\hline
\end{tabular}


TABLE 3A 2004 COLLECTION RESOURCES

\begin{tabular}{|c|c|c|c|c|c|c|c|c|c|}
\hline \multirow{3}{*}{ INSTITUTION } & \multirow{3}{*}{$\begin{array}{c}\text { STATEI I } \\
\text { NZ }\end{array}$} & \multicolumn{2}{|c|}{ MONOGRAPH VOLUMES } & \multicolumn{2}{|c|}{ NON-BOOK MATERIALS } & \multicolumn{3}{|c|}{ SERIAL VOLUMES } & \multirow{2}{*}{$\begin{array}{l}\text { ELEC. } \\
\text { SERIALS }\end{array}$} \\
\hline & & $\begin{array}{l}\text { ADDED } \\
2004\end{array}$ & $\begin{array}{c}\text { TOTAL END } \\
2004\end{array}$ & $\begin{array}{l}\text { ADDDD } \\
2004\end{array}$ & $\begin{array}{c}\text { TOTALEND } \\
2004\end{array}$ & $\begin{array}{l}\text { ADDED } \\
2004\end{array}$ & $\begin{array}{l}\text { CURRENT } \\
\text { SUBS }\end{array}$ & $\begin{array}{c}\text { TOTAL } \\
\text { END 2004 }\end{array}$ & \\
\hline & & [12] & [13] & [14] & [15] & {$[16]$} & [17] & [18] & [19] \\
\hline Adelaide College of Divinity & $\mathrm{SA}$ & 1131 & 60000 & 0 & 0 & 0 & 223 & 0 & 0 \\
\hline Alliance College of Australia & ACT & 556 & 14377 & 91 & 1254 & 0 & 48 & 147 & 0 \\
\hline Australian Lutheran College & SA & 938 & 86475 & 33 & 0 & 0 & 300 & 550 & 0 \\
\hline Bible College of New Zealand & $\mathrm{NZ}$ & 1620 & 44062 & 2 & 0 & 2 & 130 & 0 & 0 \\
\hline Bible College of South Australia & $\mathrm{SA}$ & 400 & 30267 & 74 & 1465 & 0 & 68 & 773 & 1 \\
\hline Bible College of Victoria & $\mathrm{VIC}$ & 855 & 39362 & 170 & 2516 & 3 & 135 & 160 & 0 \\
\hline Booth College of Misison & $\mathrm{NZ}$ & 742 & 11309 & 89 & 1448 & 1 & 40 & 99 & 0 \\
\hline Burleigh College & $\mathrm{SA}$ & 505 & 19000 & 18 & 485 & 7 & 50 & 140 & 2 \\
\hline Camden Theological Library & NSW & 1886 & 60644 & 15 & 382 & 0 & 120 & 500 & 0 \\
\hline Carey Baptist College & $\mathrm{NZ}$ & 583 & 33849 & 2 & 222 & 0 & 220 & 0 & 0 \\
\hline Catholic Theological College & $\mathrm{VIC}$ & 953 & 90000 & 0 & 0 & 6 & 219 & 0 & 1 \\
\hline Churches of Christ Theological College & $\mathrm{VIC}$ & 245 & 20000 & 5 & 150 & 67 & 75 & 188 & 2 \\
\hline Garden City College of Ministries & QLD & 1445 & 13742 & 6 & 658 & 1 & 11 & 36 & 6 \\
\hline Harvest Bible College & $\mathrm{VIC}$ & 4062 & 26657 & 0 & 0 & 0 & 144 & 248 & 0 \\
\hline Joint Theological Library & VIC & 2565 & 120000 & 10 & 200 & 3 & 405 & 1354 & 12 \\
\hline Kingsley College & $\mathrm{VIC}$ & 376 & 24465 & 27 & 4640 & 1 & 149 & 161 & 0 \\
\hline Moore Theological College & NSW & 7992 & 209955 & 0 & 0 & 20 & 777 & 2603 & $\underline{0}$ \\
\hline Nazarene Theological College & QLD & 772 & 35000 & 30 & 374 & 1 & 68 & 137 & 2 \\
\hline New Creation Library & SA & 280 & 8400 & 0 & 0 & 0 & 0 & 0 & 0 \\
\hline Phlair International College & $\mathrm{NZ}$ & 614 & 16970 & 190 & 2006 & 6 & 16 & 182 & 0 \\
\hline Presbyterian Theological Centre & NSW & 1828 & 32654 & 0 & 0 & 0 & 98 & 0 & 0 \\
\hline Presbyterian Theological College & VIC & 800 & 18000 & 0 & 0 & 2 & 106 & 0 & 0 \\
\hline Queensland Baptist College of Ministries & QLD & 560 & 19070 & 465 & 635 & 1 & 150 & 300 & 0 \\
\hline Ridley College & $\mathrm{VIC}$ & 1371 & 42682 & 13 & 915 & 6 & 152 & 359 & 0 \\
\hline St. Andrew's Greek Orthodox Theological & NSW & 365 & 13460 & 0 & 0 & 0 & 110 & 110 & 0 \\
\hline St. Charles Seminary & WA & 311 & 6955 & 14 & 88 & 2 & 19 & 36 & 0 \\
\hline St. Francis' Theological College & QLD & 657 & 25100 & 10 & 56 & 2 & 100 & 248 & 0 \\
\hline St. John's College & NSW & 135 & 16400 & 0 & 0 & 6 & 3 & 9000 & 2 \\
\hline St. Paschal Library & VIC & 729 & 53000 & 50 & 80 & 0 & 220 & 250 & 10 \\
\hline Sydney Missionary \& Bible College & NSW & 1626 & 25626 & 92 & 876 & 242 & 179 & 3750 & 12 \\
\hline Tabor College - Victoria & VIC & 2662 & 28730 & 57 & 1062 & 3 & 87 & 412 & 0 \\
\hline Tahlee Bible College & NSW & & & 66 & 3308 & 0 & 68 & 178 & 0 \\
\hline Talua Ministry Training Centre & VAN & 200 & 9550 & 100 & 1000 & 2 & 17 & 36 & 0 \\
\hline Trinity Theological College & WA & 839 & 13989 & 40 & 490 & 7 & 45 & 91 & 0 \\
\hline Vianney College & NSW & 238 & 14546 & 0 & 0 & 0 & 0 & 0 & 0 \\
\hline Wesley Institute & NSW & 1483 & 39703 & 20 & 1649 & 0 & 128 & 411 & 0 \\
\hline Worldview Centre for Intercultural Studies & TAS & 232 & 18924 & 123 & 4397 & 3 & 75 & 105 & 1 \\
\hline TOTAL & & 42556 & 1342923 & 1678 & 30156 & 394 & 4755 & 22564 & 51 \\
\hline AVERAGE & & 1182.11 & 37303.42 & 52.44 & 861.60 & 10.65 & 128.51 & 609.84 & 1.38 \\
\hline MEDIAN & & 757 & 25363 & 16.5 & 382 & 1 & 100 & 160 & 0 \\
\hline
\end{tabular}


TABLE 3B 2004 COLLECTION RESOURCES

\begin{tabular}{|c|c|c|c|c|c|c|c|c|c|}
\hline \multirow[t]{3}{*}{ INSTITUTION } & \multirow{3}{*}{$\begin{array}{c}\text { STATEI } \\
\text { NZ }\end{array}$} & \multicolumn{2}{|c|}{ MONOGRAPH VOLUMES } & \multicolumn{2}{|c|}{$\begin{array}{l}\text { NON-8OOK } \\
\text { MATERIALS }\end{array}$} & \multicolumn{3}{|c|}{ SERIAL VOLUMES } & \multirow{2}{*}{$\begin{array}{l}\text { ELEC. } \\
\text { SERIALS }\end{array}$} \\
\hline & & $\begin{array}{c}\text { ADDED } \\
2004\end{array}$ & $\begin{array}{l}\text { TOTAL } \\
\text { END } 2004\end{array}$ & $\begin{array}{l}\text { ADDEDA } \\
2004\end{array}$ & $\begin{array}{l}\text { TOTAL } \\
\text { END } 2004\end{array}$ & $\begin{array}{l}\text { ADDED } \\
2004\end{array}$ & & $\begin{array}{l}\text { TOTAL } \\
\text { END } 2004\end{array}$ & \\
\hline & & {$[12]$} & [13] & {$[14]$} & {$[15]$} & [16] & {$[17]$} & [18] & [19] \\
\hline Benedictine Community of New Norcia & WA & 6249 & 70000 & 68 & 332 & 0 & T & 1500 & 1 \\
\hline Carmelite Friars & VIC & 925 & 30000 & 0 & 0 & 61 & 66 & 76 & 0 \\
\hline Catholic Education Office & SA & 896 & 16301 & 170 & 4010 & 60 & 0 & 60 & 1 \\
\hline Catholic Education Office & WA & 506 & 14841 & 293 & 5865 & 12 & 97 & 105 & 4 \\
\hline Dominican Fathers & $\mathrm{VIC}$ & 1365 & 32000 & 5 & 33 & 1 & 55 & 80 & 0 \\
\hline Evangelical Library & SA & 10170 & 10548 & 1013 & 1304 & 0 & 0 & 0 & 0 \\
\hline St. Benedict's Monastery & NSW & 104 & 16150 & 0 & 75 & 40 & 0 & 1560 & 0 \\
\hline TOTAL & & 20215 & 189840 & 238 & 11619 & 174 & 218 & 3381 & 6 \\
\hline AVERAGE & & 2887.86 & 27120.00 & 79.33 & 1659.86 & 24.86 & 31.14 & 483.00 & 0.86 \\
\hline MEDIAN & & 925 & 16301 & 68 & 332 & 12 & 0 & 80 & $\mathbf{0}$ \\
\hline
\end{tabular}

TABLE 3C 2004 COLLECTION RESOURCES

\begin{tabular}{|c|c|c|c|c|c|c|c|c|c|}
\hline \multirow[t]{3}{*}{ INSTITUTION } & \multirow[t]{3}{*}{ STATE/NZ } & \multicolumn{2}{|c|}{ MONOGRAPH VOLUMES } & \multicolumn{2}{|c|}{ NON-BOOK } & \multicolumn{3}{|c|}{ SERIAL VOLUMES } & \multirow{2}{*}{$\begin{array}{l}\text { ELEC. } \\
\text { SERIALS }\end{array}$} \\
\hline & & $\begin{array}{l}\text { ADDED } \\
2004\end{array}$ & $\begin{array}{c}\text { TOTAL } \\
\text { END 2004 }\end{array}$ & $\begin{array}{l}\text { ADDED } \\
2004\end{array}$ & $\begin{array}{l}\text { TOTAL } \\
\text { END } 2004\end{array}$ & $\begin{array}{l}\text { ADDED } \\
2004\end{array}$ & $\begin{array}{l}\text { CURRENT } \\
\text { SUBS }\end{array}$ & $\begin{array}{c}\text { TOTAL } \\
\text { END } \\
2004\end{array}$ & \\
\hline & & [12] & [13] & [14] & [15] & [16] & [17] & [18] & [19] \\
\hline Australian Catholic Unievrsity - Banyo & QLD & 5944 & 141233 & 0 & 0 & 0 & 454 & 0 & 3000 \\
\hline Australian Catholic University - Signadou & ACT & 3127 & 57525 & 0 & 0 & 0 & 179 & 0 & 0 \\
\hline $\begin{array}{l}\text { Australian Catholic University - St. } \\
\text { Patrick's }\end{array}$ & VIC & 3169 & 101576 & 97 & 4490 & 31 & 278 & 750 & 0 \\
\hline $\begin{array}{l}\text { Trinity College - Leeper \& Mollison } \\
\text { Libraries }\end{array}$ & VIC & 1276 & 54000 & 98 & 157 & 0 & 131 & 337 & 0 \\
\hline University of Notre Dame & WA & 500 & 15000 & 0 & 0 & 0 & 41 & 151 & 0 \\
\hline TOTAL & & 14150 & 381381 & 97 & 7844 & 33 & 1170 & 1323 & 3000 \\
\hline AVERAGE & & 2358.33 & 63563.50 & 32.33 & 1307.33 & 5.50 & 195.00 & 220.50 & 500.00 \\
\hline
\end{tabular}


TABLE 4A 2004 LIBRARY EXPENDITURE (AU\$)

\begin{tabular}{|c|c|c|c|c|c|c|}
\hline \multirow[t]{2}{*}{ INSTITUTION } & \multirow[t]{2}{*}{$\begin{array}{l}\text { STATE } \\
\text { I NZ }\end{array}$} & MONOGRAPHS & SERIALS & $\begin{array}{l}\text { NON-BOOK } \\
\text { MATERIALS }\end{array}$ & $\begin{array}{l}\text { ELECTRONIC } \\
\text { D'BASES }\end{array}$ & TOTAL \\
\hline & & [21] & [22] & [23] & [24] & [25] \\
\hline Adelaide College of Divinity & SA & $\$ 35,138$ & $\$ 25,058$ & $\$ 0$ & $\$ 4,080$ & $\$ 64,276$ \\
\hline Alliance College of Australia & ACT & $\$ 5,497$ & $\$ 2,190$ & $\$ 217$ & $\$ 0$ & $\$ 7,904$ \\
\hline Australian Lutheran College & SA & $\$ 41,500$ & $\$ 20,000$ & $\$ 5,000$ & $\$ 5,000$ & $\$ 71,500$ \\
\hline Bible College of New Zealand & $\mathrm{NZ}$ & $\$ 31,830$ & $\$ 14,684$ & $\$ 428$ & $\$ 4,727$ & $\$ 51,669$ \\
\hline Bible College of South Australia & SA & $\$ 3,346$ & $\$ 5,700$ & $\$ 1,500$ & $\$ 1,200$ & $\$ 11,746$ \\
\hline Bible College of Victoria & VIC & $\$ 12,800$ & $\$ 9,161$ & $\$ 500$ & $\$ 0$ & $\$ 22,461$ \\
\hline Booth College of Mission & $\mathrm{NZ}$ & $\$ 11,183$ & $\$ 3,669$ & $\$ 909$ & $\$ 0$ & $\$ 15,761$ \\
\hline Burleigh College & SA & $\$ 6,000$ & $\$ 4,200$ & $\$ 45$ & $\$ 3,000$ & $\$ 13,245$ \\
\hline Camden Theological Libraryn & NSW & $\$ 44,000$ & $\$ 12,400$ & $\$ 0$ & $\$ 7,600$ & $\$ 64,000$ \\
\hline Carey Baptist College ${ }^{*}$ & $\mathrm{NZ}$ & $\$ 39,060$ & $\$ 16,740$ & $\$ 0$ & $\$ 2,790$ & $\$ 58,590$ \\
\hline Catholic Theological College & VIC & $\$ 40,900$ & $\$ 23,700$ & $\$ 0$ & $\$ 0$ & $\$ 64,600$ \\
\hline Churches of Christ Theological College & VIC & $\$ 3,861$ & $\$ 6,046$ & $\$ 0$ & $\$ 3,567$ & $\$ 13,474$ \\
\hline Garden City College of Ministries & QLD & $\$ 25,083$ & $\$ 1,300$ & $\$ 200$ & $\$ 676$ & $\$ 27,259$ \\
\hline Harvest Bible College & VIC & $\$ 17,000$ & $\$ 8,000$ & $\$ 0$ & $\$ 0$ & $\$ 25,000$ \\
\hline Joint Theological Library & VIC & $\$ 152,900$ & $\$ 64,214$ & $\$ 0$ & $\$ 0$ & $\$ 217,114$ \\
\hline Kingsley College & $\mathrm{VIC}$ & $\$ 2,738$ & $\$ 5,310$ & $\$ 300$ & $\$ 520$ & $\$ 8,868$ \\
\hline Moore Theological College & NSW & $\$ 218,006$ & $\$ 76,376$ & $\$ 13,313$ & $\$ 6,189$ & $\$ 313,884$ \\
\hline Nazarene Theological College & QLD & $\$ 11,737$ & $\$ 6,717$ & $\$ 693$ & $\$ 619$ & $\$ 19,766$ \\
\hline New Creation Library & SA & $\$ 0$ & $\$ 0$ & $\$ 0$ & $\$ 0$ & $\$ 0$ \\
\hline Phlair International College & $\mathrm{NZ}$ & $\$ 2,831$ & $\$ 562$ & $\$ 1,165$ & $\$ 0$ & $\$ 4,558$ \\
\hline Presbyterian Theological Centre & NSW & $\$ 27,974$ & $\$ 6,564$ & $\$ 0$ & $\$ 0$ & $\$ 34,538$ \\
\hline Presbyterian Theological College & VIC & $\$ 16,000$ & $\$ 8,000$. & $\$ 2,000$ & $\$ 3,000$ & $\$ 29,000$ \\
\hline Queensland Baptist College of Ministries & QLD & $\$ 20,800$ & $\$ 5,000$ & $\$ 0$ & $\$ 4,495$ & $\$ 30,295$ \\
\hline Ridley College & VIC & $\$ 37,494$ & $\$ 10,843$ & $\$ 0$ & $\$ 4,729$ & $\$ 53,066$ \\
\hline $\begin{array}{l}\text { St. Andrew's Greek Orthodox } \\
\text { Theological College }\end{array}$ & NSW & $\$ 5,849$ & $\$ 397$ & $\$ 0$ & $\$ 0$ & $\$ 6,246$ \\
\hline St. Charkes Seminary & WA & $\$ 0$ & $\$ 0$ & $\$ 0$ & $\$ 0$ & $\$ 0$ \\
\hline St. Francis' Theological College & QLD & $\$ 14,000$ & $\$ 12,200$ & $\$ 378$ & $\$ 3,174$ & $\$ 29,752$ \\
\hline St. John's College Ministry Centre & NSW & $\$ 2,500$ & $\$ 4,500$ & $\$ 0$ & $\$ 0$ & $\$ 7,000$ \\
\hline St. Paschal Library & $\mathrm{VIC}$ & $\$ 28,000$ & $\$ 15,000$ & $\$ 0$ & $\$ 0$ & $\$ 43,000$ \\
\hline Sydney Missionary \& Bible College & NSW & $\$ 49,980$ & $\$ 21,000$ & $\$ 1,658$ & $\$ 3,000$ & $\$ 75,638$ \\
\hline Tabor College - Victoria & $\mathrm{VIC}$ & $\$ 19,604$ & $\$ 12,746$ & $\$ 400$ & $\$ 4,480$ & $\$ 37,230$ \\
\hline Tahlee Bible College & NSW & $\$ 0$ & $\$ 0$ & $\$ 0$ & $\$ 0$ & $\$ 0$ \\
\hline Talua Ministry Training Centre & VAN & $\$ 0$ & $\$ 1,333$ & $\$ 1,333$ & $\$ 0$ & $\$ 2,667$ \\
\hline Trinity Theological College & WA & $\$ 47,125$ & $\$ 5,210$ & $\$ 3,828$ & $\$ 3,520$ & $\$ 59,683$ \\
\hline Vianney College & NSW & $\$ 3,000$ & $\$ 0$ & $\$ 0$ & $\$ 0$ & $\$ 3,000$ \\
\hline Wesley Institute & NSW & $\$ 0$ & $\$ 0$ & $\$ 0$ & $\$ 0$ & $\$ 41,818$ \\
\hline $\begin{array}{l}\text { Worldview Centre for Infercultural } \\
\text { Studies }\end{array}$ & TAS & $\$ 3,438$ & $\$ 2,839$ & $\$ 190$ & $\$ 27$ & $\$ 6,494$ \\
\hline TOTAL & & $\$ 981,174.19$ & $\$ 411,659.33$ & $\$ 34,057.33$ & $\$ 66,392.67$ & $\$ 1,535,101.52$ \\
\hline AVERAGE & & $\$ 26,518.22$ & $\$ 11,125.93$ & $\$ 920.47$ & $\$ 1,794.40$ & $\$ 41,489.23$ \\
\hline MEDIAN & & $\$ 14,000.00$ & $\$ 6,046.00$ & $\$ 45.00$ & $\$ 520.00$ & $\$ 27,259.00$ \\
\hline
\end{tabular}

* Exchange rate $=0.93$

^ Financial Year 03-04 
TABLE 4B 2004 LIBRARY EXPENDITURE (AU\$)

\begin{tabular}{|c|c|c|c|c|c|c|}
\hline \multirow[t]{2}{*}{ INSTITUTION } & \multirow[t]{2}{*}{$\begin{array}{c}\text { STATE } \\
\text { / NZ }\end{array}$} & MONOGRAPHS & SERIALS & $\begin{array}{l}\text { NON-BOOK } \\
\text { MATERIALS }\end{array}$ & $\begin{array}{l}\text { ELECTRONIC } \\
\text { D'BASES }\end{array}$ & TOTAL \\
\hline & & [21] & [22] & [23] & [24] & [25] \\
\hline Benedictien Community of New Norcia & WA & $\$ 13,500$ & $\$ 3,400$ & $\$ 0$ & $\$ 0$ & $\$ 16,900$ \\
\hline Carmelite Friars & VIC & $\$ 30,000$ & $\$ 3,000$ & $\$ 0$ & $\$ 0$ & $\$ 33,000$ \\
\hline Catholic Education Office & SA & $\$ 19,000$ & $\$ 1,000$ & $\$ 5,000$ & $\$ 0$ & $\$ 25,000$ \\
\hline Catholic Education Office & WA & $\$ 15,067$ & $\$ 8,909$ & $\$ 18,052$ & $\$ 12,000$ & $\$ 54,028$ \\
\hline Dominican Fathers & VIC & $\$ 13,900$ & $\$ 10,000$ & $\$ 100$ & $\$ 0$ & $\$ 24,000$ \\
\hline Evangelical Library & SA & $\$ 0$ & $\$ 0$ & $\$ 0$ & $\$ 0$ & $\$ 0$ \\
\hline St. Benedict's Monastery & NSW & $\$ 11,807$ & $\$ 3,199$ & $\$ 1,589$ & & $\$ 16,595$ \\
\hline TOTAL & & $\$ 103,274.00$ & $\$ 29,508.00$ & $\$ 24,741.00$ & $\$ 12,000.00$ & $\$ 169,523.00$ \\
\hline AVERAGE & & $\$ 14,753.43$ & $\$ 4,215.43$ & $\$ 3,534.43$ & $\$ 2,000.00$ & $\$ 24,217.57$ \\
\hline MEDIAN & & $\$ 13,900.00$ & $\$ 3,199.00$ & $\$ 100.00$ & $\$ 0.00$ & $\$ 24,000.00$ \\
\hline
\end{tabular}

TABLE 4C 2004 LIBRARY EXPENDITURE (AU\$)

\begin{tabular}{|c|c|c|c|c|c|c|}
\hline INSTITUTION & $\begin{array}{l}\text { STATE } \\
\text { I NZ }\end{array}$ & MONOGRAPHS & SERIALS & $\begin{array}{l}\text { NON-BOOK } \\
\text { MATERIALS }\end{array}$ & $\begin{array}{c}\text { ELECTRONIC } \\
\text { D'BASES }\end{array}$ & TOTAL \\
\hline & & [21] & [22] & [23] & [24] & [25] \\
\hline Australian Catholic University - Banyo* & QLD & \multirow{3}{*}{$\$ 155,000$} & \multirow{3}{*}{$\$ 77,500$} & \multirow{3}{*}{$\$ 0$} & \multirow{3}{*}{$\$ 470,000$} & \multirow{3}{*}{$\$ 702,500$} \\
\hline Australian Catholic University - Signadou* & ACT & & & & & \\
\hline Australian Catholic University - St. Patrick's ${ }^{*}$ & VIC & & & & & \\
\hline MASTERS institute & NZ & $\$ 2,250$ & $\$ 8,000$ & $\$ 250$ & $\$ 0$ & $\$ 10,500$ \\
\hline Trinity College - Leeper \& Mollison Libraries & VIC & $\$ 0$ & $\$ 0$ & $\$ 0$ & $\$ 0$ & $\$ 0$ \\
\hline University of Notre Dame & WA & $\$ 0$ & $\$ 0$ & $\$ 0$ & $\$ 0$ & $\$ 0$ \\
\hline TOTAL & & $\$ 157,250.00$ & $\$ 85,500.00$ & $\$ 250.00$ & $\$ 470,000.00$ & $\$ 713,000.00$ \\
\hline AVERAGE & & $\$ 39,312.50$ & $\$ 21,375.00$ & $\$ 62.50$ & $\$ 117,500.00$ & $\$ 178,250.00$ \\
\hline MEDIAN & & $\$ 1,125.00$ & $\$ 4,000.00$ & $\$ 0.00$ & $\$ 0.00$ & $\$ 5,250.00$ \\
\hline
\end{tabular}

* combined for all ACU campuses 
TABLE 5A 2004 INSTITUTIONAL POPULATION AND LIBRARY FACILITIES

\begin{tabular}{|c|c|c|c|c|c|c|c|c|c|}
\hline \multirow{3}{*}{ INSTITUTION } & \multirow{3}{*}{$\begin{array}{l}\text { STATE } \\
\text { I NZ }\end{array}$} & \multicolumn{2}{|c|}{ ACADEMICSTAFF } & \multicolumn{4}{|c|}{ STUDENTS } & \multirow[b]{2}{*}{$\begin{array}{l}\text { REGISTERED } \\
\text { BORROWERS }\end{array}$} & \multirow[b]{2}{*}{$\begin{array}{c}\text { TOTAL } \\
\text { SEATING } \\
\text { CAPACITY }\end{array}$} \\
\hline & & $\begin{array}{l}\text { PULL: } \\
\text { TIME }\end{array}$ & $\begin{array}{l}\text { PART:- } \\
\text { TIME }\end{array}$ & $\begin{array}{l}\text { FULL- } \\
\text { TIME }\end{array}$ & $\begin{array}{l}\text { PART- } \\
\text { TIME }\end{array}$ & $\begin{array}{l}\text { DISTANCE } \\
\text { ED. }\end{array}$ & TOTAL & & \\
\hline & & [26] & {$[27]$} & {$[28]$} & [29] & {$[30]$} & & [31] & [32] \\
\hline Adelaide College of Divinity & SA & 27 & 60 & & & & 0 & 500 & 40 \\
\hline Alliance College of Australia & ACT & 3 & 2 & 27 & 8 & 10 & & 129 & 16 \\
\hline Australian Lutheran College & SA & 12 & 13 & 72 & 55 & .428 & 555 & 1505 & 60 \\
\hline Bible College of New Zealand & NZ & 16 & 18 & 341 & 662 & 147 & & 661 & 95 \\
\hline Bible College of South Australia & SA & 2 & 17 & 18 & 64 & 121 & 203 & 217 & 27 \\
\hline Bible College of Victoria & VIC & 6 & 10 & 107 & 81 & 70 & 258 & 388 & 80 \\
\hline Booth College of Misison & $\mathrm{NZ}$ & 8 & 1 & 24 & 11 & & 35 & 257 & 53 \\
\hline Burleigh College & SA & 2 & 8 & 21 & 35 & 1 & 57 & 198 & 18 \\
\hline Camden Theological Library & NSW & 10 & 5 & 91 & 102 & & 193 & 454 & 50 \\
\hline Carey Baptist College & NZ & 10 & 11 & 202 & & 132 & 334 & 455 & 113 \\
\hline Catholic Theological College & VIC & 2 & 39 & 158 & 234 & & 392 & 355 & 72 \\
\hline Churches of Christ Theological College & $\mathrm{VIC}$ & 3 & 12 & 128 & & 0 & 128 & 210 & 15 \\
\hline Garden City College of Ministries & QLD & 4 & 4 & 23 & 28 & 0 & 51 & 368 & 18 \\
\hline Harvest Bible College & $\mathrm{VIC}$ & 9 & 5 & 169 & & 288 & 457 & 197 & 16 \\
\hline Joint Theological Library & $\mathrm{VIC}$ & 25 & 9 & 290 & 31 & 27 & 348 & 693 & 60 \\
\hline Kingsley College & $\mathrm{VIC}$ & 6 & 6 & 30 & 100 & 70 & 200 & 365 & 30 \\
\hline Moore Theological College & NSW & 20 & 16 & 291 & 157 & 0 & 448 & 550 & 33 \\
\hline Nazarene Theological College & QLD & 3 & 7 & 20 & 20 & 3 & 43 & 50 & 22 \\
\hline New Creation Library & SA & 2 & 20 & 6 & 200 & 19 & 225 & 347 & 13 \\
\hline Phlair International College & NZ & 6 & 15 & 197 & 208 & 147 & 552 & 790 & 18 \\
\hline Presbyterian Theological Centre & NSW & 4 & 18 & 42 & 71 & 10 & 123 & 130 & 36 \\
\hline Presbyterian Theological College & VIC & 4 & 6 & 0 & 0 & 0 & 0 & 0 & 18 \\
\hline Queensland Baptist College of Ministries & QLD & 4 & 6 & 50 & 86 & & 136 & 200 & 70 \\
\hline Ridley College & VIC & 7 & 8 & 73 & 157 & 0 & 230 & 405 & 80 \\
\hline St. Andrew's Greek Orthodox Theological Coll. & NSW & 19 & & 14 & 33 & 0 & 47 & 104 & 25 \\
\hline St. Charles Seminary & WA & 2 & 14 & 0 & 23 & 0 & 23 & 45 & 13 \\
\hline St. Francis' Theological College & QLD & 4 & 2 & 27 & 48 & & 75 & 242 & 32 \\
\hline St. John's College Ministry Centre & NSW & 2 & 12 & 9 & 20 & & 29 & 40 & 18 \\
\hline St. Paschal Library & $\mathrm{VHC}$ & 3 & 50 & 300 & & 0 & 300 & 150 & 40 \\
\hline Sydney Missionary \& Bible College & NSW & 11 & 10 & 170 & 315 & 0 & 485 & 506 & 70 \\
\hline Tabor College - Victoria & $\mathrm{VIC}$ & 9 & 6 & 180 & 450 & 18 & 648 & 665 & 22 \\
\hline Tahlee Bible College & NSW & 6 & 10 & 17 & 51 & 0 & 68 & 56 & 20 \\
\hline Talua Ministry Training Centre & VAN & 12 & 4 & 68 & 18 & & 86 & 120 & 24 \\
\hline Trinity Theological College & WA & 5 & 5 & 35 & 28 & & 63 & 250 & 45 \\
\hline Vianney College & NSW & 4 & 6 & 5 & 14 & 0 & 19 & 107 & 22 \\
\hline Wesley Institute & NSW & 35 & 60 & 280 & 290 & & 570 & 486 & 37 \\
\hline Worldview Centre for Intercultural Studies & TAS & 6 & 5 & 53 & 3 & 0 & 56 & 174 & 44 \\
\hline TOTAL & & 313 & 500 & 3538 & 3603 & 1491 & 7437 & 12369 & 1465 \\
\hline AVERAGE & & 8.46 & 13.89 & 98.28 & 112.59 & 55.22 & 212.49 & 334.30 & 39.59 \\
\hline MEDIAN & & 6.00 & 9.50 & 51.50 & 53.00 & 3.00 & 136.00 & 250.00 & 32.00 \\
\hline
\end{tabular}


TABLE 5B 2004 INSTITUTIONAL POPULATION AND LIBRARY FACILITIES

\begin{tabular}{|c|c|c|c|c|c|}
\hline \multirow[t]{3}{*}{ TINSTITUTION } & \multirow{3}{*}{$\begin{array}{c}\text { STATE } \\
\text { / NZ }\end{array}$} & \multicolumn{2}{|c|}{ ACADEMIC STAFF } & \multirow[b]{2}{*}{$\begin{array}{l}\text { REGISTERED } \\
\text { BORROWERS }\end{array}$} & \multirow[b]{2}{*}{$\begin{array}{c}\text { TOTAL } \\
\text { SEATING } \\
\text { CAPACITY }\end{array}$} \\
\hline & & $\begin{array}{l}\text { FULL- } \\
\text { TIMEE }\end{array}$ & $\begin{array}{l}\text { PART- } \\
\text { TIME }\end{array}$ & & \\
\hline & & [26] & [27] & [31] & [32] \\
\hline Benedictine Community of New Norcia & WA & & & & 8 \\
\hline Carmelite Friars & VIC & & & & 8 \\
\hline Catholic Education Office & SA & & & 4874 & 20 \\
\hline Catholic Education Office & WA & \multicolumn{2}{|c|}{96} & 3548 & 29 \\
\hline Dominican Fathers & $\mathrm{VIC}$ & & & 292 & 12 \\
\hline Evangelical Library & SA & & & & \\
\hline St. Benedict's Monastery & NSW & & & & 10 \\
\hline TOTAL & & 96 & & 8714 & 87 \\
\hline AVERAGE & & 96.00 & & 2904.67 & 14.50 \\
\hline MEDIAN & & 96.00 & & 3548.00 & 11.00 \\
\hline
\end{tabular}

TABLE 5C 2004 INSTITUTIONAL POPULATION AND LIBRARY FACILITIES

\begin{tabular}{|c|c|c|c|c|c|c|c|c|c|}
\hline \multirow[t]{2}{*}{ INSTITUTION } & \multirow{2}{*}{$\begin{array}{l}\text { STATE } \\
\text { I NZ }\end{array}$} & \multicolumn{2}{|c|}{ ACADEMIC STAFF } & \multicolumn{4}{|c|}{ STUDENTS } & \multirow[b]{2}{*}{$\begin{array}{c}\text { REGISTERED } \\
\text { BORROWERS } \\
\text { [31] }\end{array}$} & \multirow[b]{2}{*}{$\begin{array}{c}\text { TOTAL } \\
\text { SEATING } \\
\text { CAPACITY } \\
\text { [32] }\end{array}$} \\
\hline & & $\begin{array}{l}\text { FULL- } \\
\text { THME } \\
\text { [26] }\end{array}$ & $\begin{array}{l}\text { PART- } \\
\text { TIME } \\
\text { [27] }\end{array}$ & $\begin{array}{l}\text { FULL- } \\
\text { TIME } \\
\text { [28] }\end{array}$ & $\begin{array}{l}\text { PART- } \\
\text { TIME } \\
\text { [29] }\end{array}$ & $\begin{array}{l}\text { DIST. } \\
\text { ED. } \\
\text { [30] }\end{array}$ & TOTAL & & \\
\hline Australian Catholic University - Banyo & OLD & 75 & 25 & & & & 1800 & & 207 \\
\hline Australian Catholic University - Signadou & $\mathrm{ACT}$ & & & & & & & & \\
\hline Australian Catholic University - St. Patrick's & VIC & 117 & 105 & 2262 & 1145 & & 3407 & 3500 & 136 \\
\hline MASTERS Institute & NZ & 6. & & 50 & 0 & 50 & 100 & 70 & 17 \\
\hline Trinity College - Leeper \& Mollison Libraries & $\mathrm{VIC}$ & & & & & & 0 & 2045 & 112 \\
\hline University of Notre Dame & WA & & & & & & & & \\
\hline TOTAL & & 198 & 130 & 2312. & 1145 & 50 & 5307 & 5615 & 472 \\
\hline AVERAGE & & 66.00 & 65.00 & 1156.00 & .572 .50 & 50.00 & 1326.75 & 1871.67 & 118.00 \\
\hline MEDIAN & & 75.00 & 65.00 & 1156.00 & 572.50 & 50.00 & 950.00 & 2045.00 & 124.00 \\
\hline
\end{tabular}




\begin{tabular}{|c|c|c|c|}
\hline PRODUCT & TOTAL & PRODUCT & TOTAL \\
\hline Anchor Bible Dictionary & 2 & Let's Get lost in Jerusalem & 1 \\
\hline Aotearoa & 1 & Logos Bible Atlas & 1 \\
\hline Arthur Pink Collection & 1 & Logos Bible Software & 3 \\
\hline ATLA Religion Index & 26 & Marriage, Family and Christian Counseling & 1 \\
\hline ATLAS & 6 & Martin Luther & 1 \\
\hline Australia's Religious Communities & 16 & Master Christian Library Discovery Edition & 3 \\
\hline Baker Digital Reference Library & 1 & Nelson's Electronice Bible Reference Library & 1 \\
\hline Bible Atlas & 1 & New Dictionary of New Testament Theology & 1 \\
\hline Bible in English & 1 & New Testament Abstracts (NTA) & 2 \\
\hline Bible Software 2.0 & 1 & Old Testament Abstracts (OTA) & 2 \\
\hline BibleWorks & 1 & Operation World & 2 \\
\hline Book of Worship & 1 & Phi Greek Documentary Texts & 1 \\
\hline Bookshelf '95 & 1 & Proquest Religion Database & 15 \\
\hline Catechism \& Scripture & 1 & Religion and Philosophy Coillection & 15 \\
\hline Catholic Documents & 1 & Religious and Theological Abstracts (RTA) & 5 \\
\hline Catholic Periodical \& Literature Index (CPLI) & 7 & Research Methods for Ministry and Missions & 1 \\
\hline Changing Society, Changing Religion & 1 & Routledge Encyclopedia of Philosophy & 1 \\
\hline Christian History Interactive & 2 & SAGE Digital Library & 2 \\
\hline Christian Library & 1 & Spurgeon Collection & 2 \\
\hline Dead Sea Scrolls & 1 & The Message & 1 \\
\hline Desktop Bible & 1 & Theological Journal Library & 2 \\
\hline Dictionary of New Testament Theology & 1 & TLG & 2 \\
\hline Dictionary of Old Testament Theology & 1 & Trends 2100 & 1 \\
\hline Early Church Fathers & 1 & Tresures Imperials & 1 \\
\hline Encarta ‘97 & 1 & Unlocking Time: Bible Chronology & 1 \\
\hline Encyclopedia Britannica & 3 & Welcome to the Catholic Church & 1 \\
\hline Essential IVP Reference Collection & 1 & Westminster Confessions & 1 \\
\hline Expositor's Bible Commentary & 1 & Westminster Journal & 1 \\
\hline Gramcord & 1 & Whole Bible Classic Sermon Collection & 1 \\
\hline Greek Tutor & 2 & Works of Cornelius Van Til & 1 \\
\hline Hebrew Tutor & 2 & World Atlas & 1 \\
\hline Japan 2000 & 1 & World Christian Trends AD30-AD2200 & 1 \\
\hline John Calvin & 3 & World of Islam & 7 \\
\hline John Owen & 1 & World Religions & 1 \\
\hline Justice and Equity & 1 & Worldwide Prayer Guide & 1 \\
\hline Lest we Forget: a history of the Holocaust & 1 & $\begin{array}{l}\text { WorldVue: the great commission map } \\
\text { collection }\end{array}$ & 1 \\
\hline
\end{tabular}

